\title{
A Near Infrared Camera Refrigerated by Two Stirling Machines - an Alternative to Robotic Telescopes
}

\author{
José K. Ishitsuka I. ${ }^{1}$, Takehiko Wada ${ }^{2}$, Fumihiko Ieda ${ }^{3}$, \\ Noritaka Tokimasa ${ }^{4}$, Takehiko Kuroda ${ }^{4}$, Masaki Morimoto ${ }^{4}$, \\ Takeshi Miyaji ${ }^{5}$, Toshihiro Omodaka ${ }^{3}$, Munetaka Ueno ${ }^{1}$, \\ Wataru Hasegawa ${ }^{3}$, Shin-ya Narusawa ${ }^{4}$, and Yoshifumi Waki ${ }^{6}$ \\ ${ }^{1}$ Department of Earth Science and Astronomy, University of Tokyo, \\ Komaba, Tokyo 153-8902 \\ 2 Institute of Space and Astronautical Science, Sagamihara, Kanagawa \\ 229-8510 \\ ${ }^{3}$ Faculty of Science, Kagoshima University, Kagoshima 890-0065 \\ ${ }^{4}$ Nishi-Harima Astronomical Observatory, Sayo, Hyogo 679-5313 \\ ${ }^{5}$ Nobeyama Radio Observatory, National Astronomical Observatory of \\ Japan, Minami-saku, Nagano 384-1305 \\ ${ }^{6}$ Association of Amateur Astronomers, Nishi-Harima Astronomical \\ Observatory.
}

\begin{abstract}
We have developed and tested a new near infrared camera equipped with a $512 \times 512 \mathrm{PtSi} \mathrm{CCD}$ and cooled by two independent Stirling Cycle refrigerators. The camera, installed on the $60 \mathrm{~cm}$ reflector telescope of the Nishi-Harima Astronomical Observatory (NHAO) since April 2000, has begun regular observations toward infrared objects. Since the reasonable cost and lower maintenance needs of the camera make it more attractive, we introduce it as an alternative to robotic telescopes.
\end{abstract}

\section{Introduction}

We took four years to develop the near infrared camera NIHCOS (Ishitsuka et al. in preparation) that uses a $512 \times 512 \mathrm{PtSi}$ CCD proved for astronomical applications. The project is supported by the Hyogo Prefecture Nishi-Harima Astronomical Observatory, the University of Kagoshima, the National Astronomical Observatory of Japan, the Institute of Space and Astronautical Science and the University of Tokyo. NHAO is a public observatory managed by a local government, the Hyogo Prefecture. The main objective of the observatory is education and popularization of Astronomy within the general public. But it also performs scientific oriented observations.

\section{Observations and Preliminary Results}

Regular observations were begun from April 24th of 2000, we began infrared photometry of the semi-regular variable star R Crateris. Simultaneously, the University of Kagoshima with the $6 \mathrm{~m}$ radio telescope performed the monitoring 

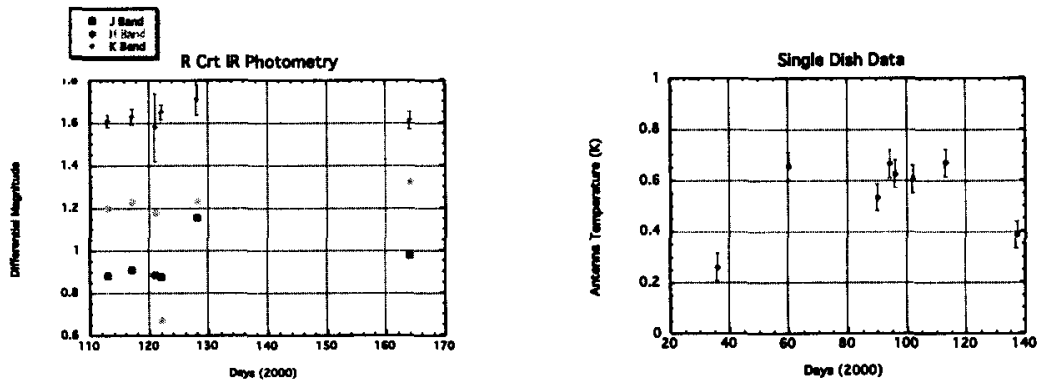

Figure 1. Simultaneous IR photometry and microwave measurements of $\mathrm{R}$ Crt

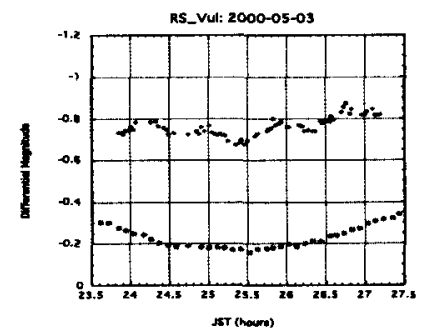

Figure 2. RS Vul, V band and $\mathrm{H}$ band simultaneous photometry

of water vapor maser at $22 \mathrm{GHz}$ (see Figure 1). The aim of the observations is to find the correlation between intensities of maser and dust emission and to determine the water maser radiation mechanism. On the other hand, the NHAO supports a group of amateur astronomers, and one of the members, Y. Waki, performed V band photometry of the binary star RS Vul, while infrared photometry was in progress. The equipment used for $\mathrm{V}$ band observations is a $7.5 \mathrm{~cm}$ refractor telescope with a ST-7 CCD. The results of the simultaneous photometry are presented in Figure 2.

\section{Conclusion}

We successfully tested our infrared camera system and made photometric measurements, also imaged planets, galaxies, globular clusters, planetary nebulae, etc. The camera system is a prototype and could be an alternative to fully automated or robotic telescopes.

Acknowledgments. The author gratefully acknowledges the financial assistance from the Japanese Foundation for Promotion of Astronomy (Travel grant) and the IAU grants that made it possible to attend the meeting. 